\title{
Poly(dimethyl siloxane) surface modification with biosurfactants isolated from probiotic strains
}

\author{
S. Pinto, ${ }^{1}$ P. Alves, ${ }^{1}$ A. C. Santos, ${ }^{2}$ C. M. Matos, ${ }^{2}$ B. Oliveiros, ${ }^{2}$ S. Gonçalves, ${ }^{3}$ E. Gudiña, ${ }^{3}$ \\ L. R. Rodrigues, ${ }^{3}$ J. A. Teixeira, ${ }^{3}$ M. H. Gil ${ }^{1}$ \\ ${ }^{1}$ Department of Chemical Engineering, University of Coimbra, Polo II, Pinhal de Marrocos, 3030-790 Coimbra, Portugal \\ ${ }^{2}$ Institute of Biophysics/Biomathematics, Biomedical Institute of Research in Light and Image (IBILI), Faculty of Medicine, \\ University of Coimbra \\ ${ }^{3}$ IBB_-Institute for Biotechnology and Bioengineering, Centre of Biological Engineering, Universidade do Minho, \\ 4710-057 Braga, Portugal
}

Received 14 January 2011; accepted 19 April 2011

Published online in Wiley Online Library (wileyonlinelibrary.com). DOI: 10.1002/jbm.a.33146

\begin{abstract}
Depending on the final application envisaged for a given biomaterial, many surfaces must be modified before use. The material performance in a biological environment is mainly mediated by its surface properties that can be improved using suitable modification methods. The aim of this work was to coat poly(dimethyl siloxane) (PDMS) surfaces with biosurfactants (BSs) and to evaluate how these compounds affect the PDMS surface properties. BSs isolated from four probiotic strains (Lactococcus lactis, Lactobacillus paracasei, Streptococcus thermophilus A, and Streptococcus thermophilus B) were used. Bare PDMS and PDMS coated with BSs were characterized by contact angle measurements, infrared spectroscopy (ATR-FTIR), X-ray photoelectron spectroscopy (XPS), and atomic force microscopy (AFM). The influence of the surface modifications on the materials blood compatibility was studied through thrombosis and hemolysis assays. The
\end{abstract}

cytotoxicity of these materials was tested against rat peritoneal macrophages. AFM results demonstrated the successful coating of the surfaces. Also, by contact angle measurements, an increase of the coated surfaces hydrophilicity was seen. Furthermore, XPS analysis indicated a decrease of the silicon content at the surface, and ATR-FTIR results showed the presence of BS characteristic groups as a consequence of the modification. All the studied materials revealed no toxicity and were found to be nonhemolytic. The proposed approach for the modification of PDMS surfaces was found to be effective and opens new possibilities for the application of these surfaces in the biomedical field. ( 2011 Wiley Periodicals, Inc. J Biomed Mater Res Part A: 00A: 000-000, 2011.

Key Words: poly(dimethyl siloxane), biosurfactants, plasma, surface modification

\section{INTRODUCTION}

Recently, the use of silicone elastomers based upon poly (dimethyl siloxane) (PDMS) has been increasing. PDMS materials are widely used in the biotechnology field, as microfluidic devices, in microcontact printing technology, biocompatible devices (e.g., contact lenses and voice prosthesis) and drug-delivery systems. This is a result of their attractive properties, namely, (i) high gas permeability, (ii) stability and optical transparency, (iii) good flexibility and mechanical stability, (iv) nontoxicity and biocompatilibity, (v) stability toward heat and chemicals, (vi) low curing temperature, (vii) moldability, and (viii) low cost. ${ }^{1,2}$

For many biomedical applications, the extensive hydrophobicity of the PDMS surfaces is disadvantageous and often demands further modifications in order to enable their use. Hydrophobic surfaces tend to adsorb significant amounts of proteins from the surrounding biological environment, followed by microbial adhesion and biofilm formation. ${ }^{3,4}$ Surface free energy, surface charge, and the hydrophobic/hydrophilic ratio are the features that most influence the material performance and microbial adhesion. ${ }^{5}$

Many studies have reported the hydrophilization of PDMS as a way to improve the material biocompatibility. ${ }^{6}$ Several techniques, involving physical or chemical treatments, or a combination of both, have been reported to modify the surface properties of PDMS and improve its wettability. ${ }^{7}$ Among these techniques, plasma, ultraviolet light, and corona discharge have been the more commonly used. Nevertheless, these modifications are only temporary, as the surface eventually recovers its native hydrophobic state. $^{8,9}$ The hydrophobic recovery in the PDMS surfaces can be due to the thermodynamically favorable orientation of the functional groups, reorientation of surface silanol groups into the bulk polymer, and the migration of lower molecular weight PDMS chains to the surface. ${ }^{10}$

One effective approach to avoiding microbial adhesion on biomaterials is the modification of the surface properties by conditioning it with biosurfactants (BS). These compounds 
may interact with interfaces, changing the surface properties and consequently affecting the adhesion and detachment of microorganisms. ${ }^{5}$

BSs are surface-active compounds that are synthesized by microorganisms and comprise a wide range of structures, such as glycolipids, lipopeptides, polysaccharide-protein complexes, phospholipids, fatty acids, and neutral lipids. ${ }^{11,12}$ These compounds present several advantages over their synthetic counterparts, namely low toxicity, biodegradability, chemical diversity, effectiveness under extreme environmental conditions, surface activity, emulsifying ability, antimicrobial, and antiadhesive properties. Furthermore, BS can be obtained from renewable substrates, reducing the manufacturing costs and increasing the commercial viability. Thus, interest in these compounds has increased significantly. Several applications have been reported for BS in petroleum, pharmaceutical and food processing industries. Nevertheless, only few studies were conducted on their possible application in the biomedical field. ${ }^{11-13}$ Therefore, the main goal of this work was to modify PDMS surfaces with BSs isolated from probiotic strains in order to improve some of its properties, thus enabling its use as a biomaterial.

In the present study, the modification of bare PDMS surfaces was performed by argon plasma treatment, followed by coating with different BSs. The morphology of the modified surfaces was studied by atomic force microscopy (AFM). Moreover, the extent of surface modification, namely the surface elemental composition, was assessed by X-ray photoelectron spectroscopy (XPS). Also, ATR-FTIR analysis was conducted to determine the nature of the chemical groups that were present at the surface. Furthermore, contact angles were measured to assess changes in hydrophobocity for the different modified surfaces. Finally, blood compatibility (thrombosis and hemolysis) and cytotoxicity (against rat peritoneal macrophages) assays were performed to evaluate the biocompatibility of the modified PDMS surfaces.

\section{MATERIALS AND METHODS Reagents}

Sylgard ${ }^{\circledR} 184$ (Down Corning), a PDMS kit containing two parts, a liquid silicon rubber base and a curing agent, was purchased from DOW-Corning. BSs from probiotic strains, namely Lactococcus lactis, Lactobacillus paracasei, Streptococcus thermophilus A, and Streptococcus thermophilus B, were obtained according to the procedures described in Refs. ${ }^{14-17}$ and kindly provided by the Institute for Biotechnology and Bioengineering, Universidade do Minho (Portugal).

\section{PDMS: Synthesis and surface modification}

PDMS prepolymer and curing agent $\left(\right.$ Sylgard $^{\circledR} 184$, Dow Corning) were mixed at the ratio of $10: 1$ by mass, stirred thoroughly, and then degassed under vacuum. The mixture was poured into Petri dishes $(0.5 \mathrm{~mm}$ of thickness) and cured for $4 \mathrm{~h}$ at $65^{\circ} \mathrm{C}$. Following curing, PDMS films were washed thoroughly with acetone.

To create reactive sites for subsequent attachment of the BSs, PDMS films were treated with argon plasma (Femto System) for $2 \mathrm{~min}$, a chamber pressure of $0.6 \mathrm{mbar}$, and power plasma of $100 \mathrm{~W}$. Low pressure plasma manufactured by Diener Electronics was used. Afterward, the films were dipped into a $1 \%(\mathrm{w} / \mathrm{v})$ BS aqueous solution at room temperature for $24 \mathrm{~h}$. This procedure was performed for each of the four studied BSs.

\section{Water Contact Angle Measurements}

Static contact angle measurements were obtained using OCA 20 from Dataphysics to determine the effect of BSs on surface wettability. ${ }^{18,19}$ Water contact angle measurements were performed at room temperature using the sessile drop method. The reported angles consist of an average of seven independent measures.

Attenuated Total Reflection Fourier Transform Infrared Fourier transform infrared (IR) spectroscopy in the attenuated total reflection mode (FTIR-ATR) was used to assess the changes on the surfaces after modification. Spectra were record on a JASCO FTIR-4200 spectrometer. All spectra were obtained from 180 scans with a resolution of $4 \mathrm{~cm}^{-1}$ in the range of $550-4000 \mathrm{~cm}^{-1}$.

\section{X-ray Photoelectron Spectroscopy}

X-ray photoelectron spectroscopy (XPS) spectra were obtained using a VGS ESCALAB 200A spectrometer with an $\mathrm{Al} \mathrm{K \alpha} \mathrm{X}$-ray source. The operation conditions were set to $15 \mathrm{kV}$. The core-level signals were obtained at a photoelectron take-off angle of $0^{\circ}$ regarding the sample surface. The binding energies were referred to the C1s-binding energy of the hydrocarbon of the PDMS (285 eV). The curve fitting of the C1s peak was carried out with the XPSpeak 4.1 software using a combination of Gaussian and Lorentzian functions. ${ }^{20,21}$

\section{Atomic Force Microscopy}

The morphology of the surfaces was studied by atomic force microscopy (AFM). AFM measurements were carried out with a NanoScope Iva Veeco Metrology. Tapping-mode scans were performed (scan size $4.0 \mu \mathrm{m}$ and scan rate $1.0 \mathrm{~Hz}$ ) at ambient conditions. The average roughness $\left(R_{\mathrm{a}}\right)$ was calculated directly from $700 \times 700 \mathrm{~nm}^{2}$ sized height AFM images. The surface roughness was measured at three different positions on each sample.,

\section{Hemocompatibility}

The biological response to bare and to BS-modified PDMS was studied through thrombogenicity and hemolysis assays using rabbit blood with acid-citrate-dextrose (ACD).

Thrombogenicity assay. The four biosurfcatant-PDMS modified surfaces were used, and a control sample, corresponding to bare PDMS, was included. The extent of thrombogenicity was evaluated through a gravimetric analysis, an adaptation of the method described by Imai and Nose. ${ }^{23}$ Contact times of 20 and $40 \mathrm{~min}$ were used in these experiments.

Before testing, the films were kept in contact with phosphate-buffered saline solution (PBS, $0.01 \mathrm{M}$, and $\mathrm{pH} 7.4$ ) at 


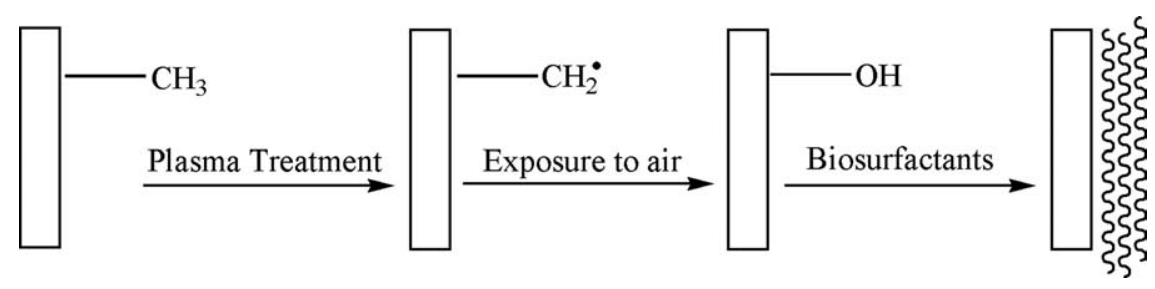

FIGURE 1. Schematic representation of the biosurfactant coating procedure onto the PDMS surface.

$37^{\circ} \mathrm{C}$ for $24 \mathrm{~h}$. Briefly, $250 \mu \mathrm{L}$ of blood with ACD anticoagulant was placed on the surface of each film (one film, in triplicate, for each contact time). Afterward, $25 \mu \mathrm{L}$ of $0.1 \mathrm{M}$ $\mathrm{CaCl}_{2}$ was added to start the clotting process, and then films were incubated at $37{ }^{\circ} \mathrm{C}$. The clotting process was stopped by adding distilled water $(5.0 \mathrm{~mL})$ after 20 and $40 \mathrm{~min}$, respectively. Clots were fixed using $36.5 \%(\mathrm{w} / \mathrm{w})$ formaldehyde solution $(5.0 \mathrm{~mL})$. Then, they were dried at $37^{\circ} \mathrm{C}$, until constant weight. This assay includes a positive $(+)$ control (glass Petri dish). The thrombosis degree was calculated and expressed as a percentage in relation to the positive assay, as described elsewhere. ${ }^{23,24}$

Hemolysis assay. Direct hemolysis contact tests were performed according to ISO 10993-4:20002 and ASTM F756-00 standards ${ }^{25,26}$ for the bare and BS-modified PDMS. Materials were incubated with diluted ACD blood (1 $\mathrm{mL}$ of diluted blood for each $7 \mathrm{~cm}^{2}$ of material), during $4 \mathrm{~h}$, at $37^{\circ} \mathrm{C}$, under static conditions. Positive $(+)$ (total hemolysis) and negative ( - ) controls were obtained by adding rabbit blood to distilled water and PBS solution, respectively. After centrifugation at $750 \times g(15 \mathrm{~min})$, the hemoglobin released by hemolysis was measured by the cyanmethemoglobin method described elsewhere. ${ }^{23,24,26}$ The supernatant absorbance, at $540 \mathrm{~nm}$, corresponds to the hemoglobin present; thus it is an indication of red cells lysis.

The hemolysis degree was calculated and expressed as a percentage in relation to the hemoglobin concentration in the positive control, after subtracting the negative control. ${ }^{24}$

According to the ASTM F 756-00 standard, materials are classified as nonhemolytic if the hemolysis degree is less than $2 \%$, slightly hemolytic if the hemolysis degree is comprised between 2 and $5 \%$ and hemolytic for values above $5 \%{ }^{26}$

\section{Viability and cytotoxicity assays}

Peritoneal macrophages were harvested from Wistar rats (Charles River, Spain) using aseptic conditions in a laminar flow chamber and cultured according to the protocol established by the Biophysics/Biomathematics Institute (Faculty of Medicine of the University of Coimbra) that was optimized from the Weir's method. ${ }^{27,28}$ Complete RPMI 1640 medium was used as culture medium in sterile conditions at $37^{\circ} \mathrm{C}$ and $5 \% \mathrm{CO}_{2}$.

First, the viability of the macrophages was evaluated based on their ability to phagocyte the trypan blue dye, also enabling cell counting in a Neubauer chamber with an inverted optical microscope. Macrophages' viability and cytotoxicity were then studied using the 3-(4,5-dimethylthriazol-2,5-diphenyltetrazolium bromide) (MTT) assay at 3 and 5 days of incubation in the referred conditions. ${ }^{3,28}$ Triplicate samples were assayed for each incubation time.

After 3 or 5 days, the culture medium was removed and $270 \mu \mathrm{L}$ of new sterile complete RPMI 1640 medium plus 30 $\mu \mathrm{L}$ of MTT solution were added to each well and incubated for $3 \mathrm{~h}\left(37^{\circ} \mathrm{C}\right.$ and $\left.5 \% \mathrm{CO}_{2}\right)$. Next, the whole solution was removed, $300 \mu \mathrm{L}$ of isopropanol acid was added, and the cells were maintained for $15 \mathrm{~min}$ at room temperature to ensure that all the formazan crystals were dissolved. Absorbance, which is directly proportional to cell viability, depicting the mitochondrial activity, was read at $570 \mathrm{~nm}$ using a MicroELISA SLT (SLT SPECTRA, Lab Instruments). ${ }^{3,28}$

Statistical analysis was performed using parametric tests to compare the average values. A two-way ANOVA was applied with post hoc comparisons by the Tukey test whenever significant differences were achieved by the previous test, as long as the dependent variable data were normally distributed. The nonparametric test of Kruskal-Wallis was also used with multiple comparisons by the Mann-Whitney test revised by Benferroni to compare all samples through time (days 3 and 5). Statistical inference was interpreted at $5 \%$ significance level. Statistica ${ }^{\circledR}$ (version 8) software was used in this analysis. ${ }^{29}$

\section{RESULTS AND DISCUSSION}

\section{Surface modification}

In a low pressure system, high discharges of gas molecules lead to the formation of ionized species, namely electrons that have enough kinetic energy to break covalent bonds in solid surfaces. ${ }^{30}$ In this case, the argon plasma treatment leads to the breakage of $\mathrm{C}-\mathrm{H}$ bonds of the methylene groups and consequently produces carbon radicals in the PDMS surface. After a given exposure time to air, the surface undergoes oxidation, leading to the formation of peroxide and hydroxyl peroxide species, among other oxidized carbon species. ${ }^{10,30}$ These reactive species can bond with the BSs that stay adsorbed at the surface, according to the scheme presented in Figure 1.

\section{Contact Angles}

Contact angle measurements were carried out on the bare PDMS surface and on the plasma PDMS surface that had been treated with BSs coatings. Water contact angles were measured after surface modification, thoroughly washed and dried. Table I shows the contact angles measured for 
TABLE I. Water Contact Angles Obtained for the Bare and Biosurfactant-Modified PDMS

\begin{tabular}{lcccr}
\hline PDMS & Modification 1 & Modification 2 & Modification 3 & Modification 4 \\
\hline $112.0 \pm 1.3$ & $44.0 \pm 3.3$ & $43.7 \pm 3.8$ & $51.4 \pm 3.3$ & $49.3 \pm 3.5$ \\
\hline
\end{tabular}

Biosurfactant-modified PDMS: Modification 1, PDMS plus biosurfactant from Streptococcus thermophilus A; Modification 2, PDMS plus biosurfactant from Streptococcus thermophilus B; Modification 3, PDMS plus biosurfactant from Lactococcus lactis; Modification 4, PDMS plus biosurfactant from Lactobacillus paracasei. Results represent the average of seven independent measures \pm standard deviation.

the different modifications performed. PDMS is very hydrophobic (contact angle of $112.0 \pm 1.3$ ), with apolar groups (methyl) at the surface, being the water contact angle very high due the weak interactions that are established between water and the PDMS surface (Van Der Waals interactions). ${ }^{3,10}$ BSs are large molecules that have polar and apolar groups. ${ }^{11}$ After adsorption, some of the polar groups of the BSs stay at the surface, and, therefore, the water contact angle decreases $\left(\sim 60^{\circ}\right.$ when compared with the bare PDMS) due dipole-dipole interactions. These results suggest that the surface of PDMS was efficiently modified.

Rodrigues and coworkers have determined the contact angles of untreated silicone rubber and BSs adsorbed silicone rubber and achieved similar values: $109 \pm 2$ and $48 \pm 7$, respectively. ${ }^{31}$

\section{Attenuated Total Reflection Fourier Transform Infrared}

The FTIR method has been widely used to characterize the surface chemical groups, because infrared (IR) transmission spectra present peak shapes characteristic of specific chemical bonds. ${ }^{20}$ Comparison of the spectra peaks and corresponding chemical groups, between bare PDMS surfaces and BS-modified PDMS surfaces, is shown in Figure 2 and Table II. The ATR-FTIR spectra obtained for the different modified surfaces showed the characteristic peaks of the BSs, in addition to the regular peaks of PDMS, thus confirming the modification of the surface. The presence of a $3200-$ $3500 \mathrm{~cm}^{-1}$ peak is a clear indication of the $\mathrm{OH}$ and $\mathrm{NH}$ groups present in glycoproteins (structures proposed for the BSs obtained from L. lactis and L. paracase $i^{14,15,17}$ ). For the PDMS surfaces modified with the BSs from $S$. thermophilus $\mathrm{A}$ and $S$. thermophilus $\mathrm{B}$, this peak is not evident. A peak at 1650 and one at $1520 \mathrm{~cm}^{-1}$, corresponding to $\mathrm{C}=0$ stretching of carbonyl groups and $\mathrm{NH}$ bending (peptide linkage), can be observed in the spectra corresponding to these surfaces. The peak corresponding to $\mathrm{C}-\mathrm{O}$ stretching in sugars (1000-1200 $\mathrm{cm}^{-1}$ ) was found to overlap the one corresponding to the asymmetric $\mathrm{Si}-\mathrm{O}-\mathrm{Si}$ stretching in [- $\left.\left(\mathrm{CH}_{2}\right)_{2} \mathrm{Si}-\mathrm{O}-\right]_{x}\left(1000-1100 \mathrm{~cm}^{-1}\right)$. Previously, a glycolipid-like structure has been proposed for the BSs obtained from $S$. thermophilus strains. ${ }^{16,32}$ Nevertheless, as the BSs used in the current work had not been purified (crude BSs), it is possible that other functional groups, such as the peptide characteristic groups, were also at the surface.

\section{X-ray photoelectron spectroscopy}

The X-ray photoelectron spectroscopy (XPS) technique was used to determine the elemental composition of the surfaces and also to provide information concerning chemical bonding. The results presented in Table III clearly demonstrate a change in the elemental composition of the PDMS surface after modification with BSs, namely the decrease of the Si content and the appearance of nitrogen. The appearance of nitrogen is in accordance with the results observed from the ATR-FITR analysis. The silicon atom content decreases while the carbon and oxygen contents slightly increase,

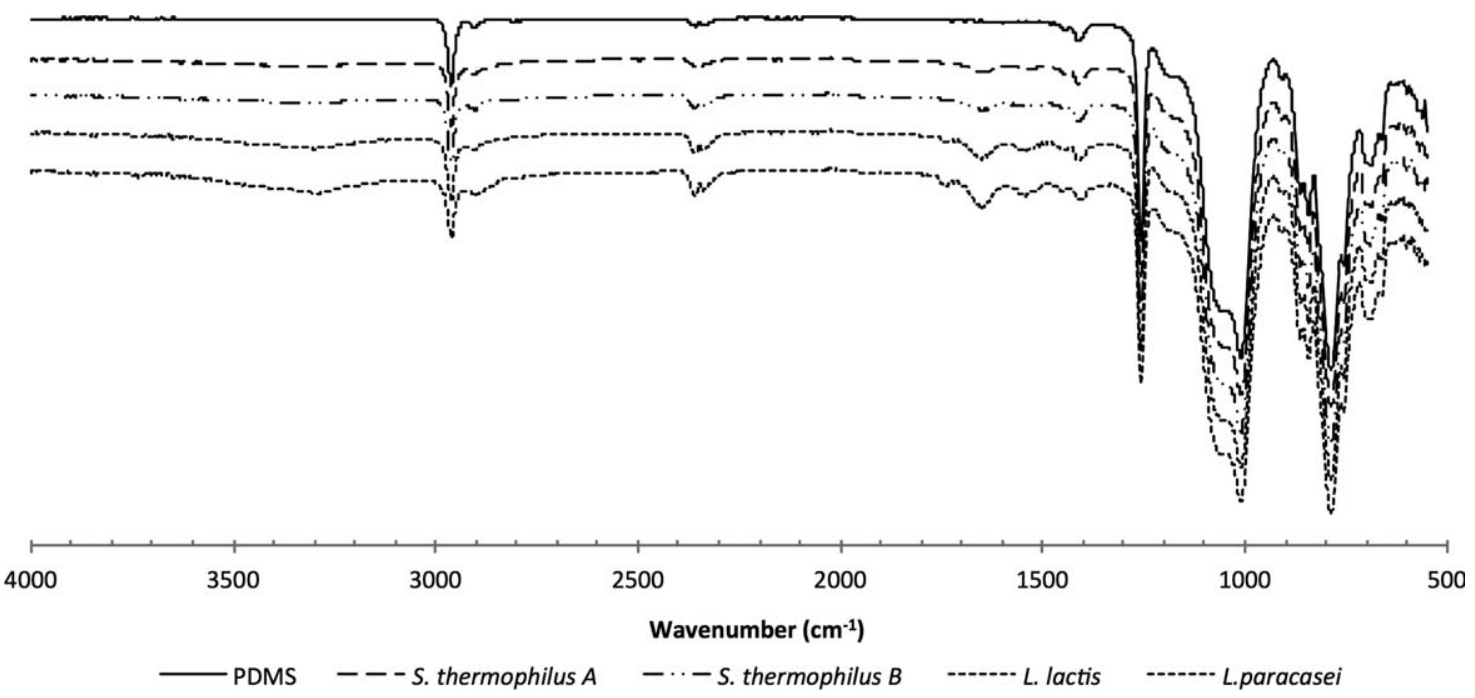

FIGURE 2. ATR-FTIR spectra from bare and biosurfactant-modified PDMS surfaces. PDMS, bare PDMS; S. thermophilus A, PDMS modified with biosurfactant from Streptococcus thermophilus A; S thermophilus B, PDMS modified with biosurfactant from Streptococcus thermophilus B; L. lactis, PDMS modified with biosurfactant from Lactococcus lactis; L. paracasei, PDMS modified with biosurfactant from Lactobacillus paracasei. 
TABLE II. Correspondence Between IR Spectra and Functional Groups Detected at the Surfaces of Bare and Biosurfactant-Modified PDMS

\begin{tabular}{lc}
\hline Group or functionality & Regions $\left(\mathrm{cm}^{-1}\right)$ \\
\hline Bare PDMS & \\
$\mathrm{C}-\mathrm{H}$ (stretching) of $\mathrm{CH}_{3}$ & $2900-3000$ \\
$\mathrm{CO}_{2}$ & 2350 \\
Asymmetric $-\mathrm{CH}_{3}$ deformation & 1460 \\
Symmetric $-\mathrm{CH}_{3}$ deformation & 1260 \\
Asymmetric $\mathrm{Si}-\mathrm{O}-\mathrm{Si}$ stretching in & $1000-1150$ \\
{$\left[-\left(\mathrm{CH}_{2}\right)_{2} \mathrm{Si}-\mathrm{O}-\right]_{X}$ vibrations of } & \\
$-\left(\mathrm{CH}_{2}\right)-$ in $\mathrm{Si}-\left(\mathrm{CH}_{2}\right)_{2}-\mathrm{Si}$ & \\
$-\mathrm{CH}_{3}$ rocking and $\mathrm{Si}-\mathrm{C}$ stretching in $\mathrm{Si}-\mathrm{CH}_{3}$ & $785-850$ \\
Biosurfactant-modified $P D M S$ & \\
$\mathrm{OH}$ and NH stretching & $3200-3600$ \\
$\mathrm{C}-\mathrm{H}$ (stretching) & $2800-3000$ \\
$\mathrm{CO}{ }_{2}$ & 2350 \\
$\mathrm{C}=\mathrm{O}$ (stretching) & 1725,1675 \\
$\mathrm{~N}-\mathrm{H}$ (bending) & 1520 \\
$\mathrm{CH}$ (scissor) & 1460 \\
$\mathrm{C}-\mathrm{O}$ sugars (stretching) & $1000-1300$ \\
\hline
\end{tabular}

suggesting the presence of a different layer at the surface, richer in carbon, oxygen, and nitrogen. These results also suggest that the layer present at surface is very thin or that the percentage of surface coverage with BSs is low, because the Si content, although decreased in the modified surfaces, is still substantial. Furthermore, the presence of chloride unit in these surfaces is probably a consequence of some contamination occurring during the surface modification procedure or the BS isolation, because it was not expected that the chloride species could be present on the surfaces.

The XPS spectra of $\mathrm{C}-1 \mathrm{~s}$ for all the studied surfaces are presented in Figure 3. The $\mathrm{C}-1 \mathrm{~s}$ core level spectrum is curved fitted with one peak, at a binding energy of $285 \mathrm{eV}$, assigned to the methyl carbons present at PDMS. The presence of BSs can be deduced from the appearance of the C-1s component, located at about $285 \mathrm{eV}$, associated with $\mathrm{C}-\mathrm{C}, \mathrm{C}-\mathrm{H}$, and $\mathrm{C}-\mathrm{Si}, 286.5 \mathrm{eV}$ attributable to the $-\mathrm{C}-\mathrm{O}-\mathrm{C}-$ and $\mathrm{C}-\mathrm{OH}$ species, as well as to the presence of the $\mathrm{C}$-1s core-level signal, at about $288.5 \mathrm{eV}$, associated with ester and peptide linkage (COOC and $\mathrm{CONH}$ ). ${ }^{8,20}$ The main peak observed, for all modifications, is associated to hydrocarbon bonds, suggesting that the $\mathrm{C}-\mathrm{C}$ and $\mathrm{C}-\mathrm{H}$ are also present in the BSs. Again, these results are in accordance with the previously obtained results by ATR-FTIR analysis.

\section{Atomic Force Microscopy}

The use of the NanoScope software enabled the evaluation of the surface roughness to be carried out for each modification. According to Figure 4, the surface of bare PDMS is homogeneous and smooth, with a very small average roughness value $(0.37 \pm 0.03 \mathrm{~nm})$. The presence of BSs increased the surface roughness [Fig. 4(b-e)], with the formation of domains, due to the aggregation of BSs at the surface. As these domains are not present on the bare PDMS surface, it was possible to verify that the proposed modifications occurred. Also, Figure 4 shows that the $S$. thermophilus A coating leads to the most heterogeneous coating, confirmed by the surface roughness value, $R_{\mathrm{a}}=3.86 \pm 0.87 \mathrm{~nm}$, while the PDMS surface modified with BS from L. paracasei promotes the most uniform and smooth surface, with an $R_{\mathrm{a}}=$ $2.74 \pm 0.33 \mathrm{~nm}$. Moreover, the L. lactis coating leads to a surface roughness of $3.23 \pm 0.65 \mathrm{~nm}$. The $S$. thermophilus B coating leads to a value of $3.37 \pm 0.51 \mathrm{~nm}$. A previous study of Pluronic ${ }^{\circledR}$ F-68 modified PDMS surface, produced similar values of the roughness for bare PDMS $(0.49 \pm 0.03$ $\mathrm{nm})$, also showed an increase when coated with a synthetic surfactant $(4.79 \pm 0.11 \mathrm{~nm}) .^{3}$

\section{Hemocompatibility}

As previously described, the biological responses of bare PDMS surfaces and BS modified PDMS surfaces, namely through thrombogenicity and hemolysis assays, were studied.

Thrombogenicity assay. The thrombogenicity results are given in Figure 5. The thrombogenic properties were determined by measuring the weight of the thrombus formed. The thrombus formation represents a measure of blood compatibility of a given material. ${ }^{11}$ The results show that for contact times with blood of 20 and $40 \mathrm{~min}$, the BSscoated surfaces present a lower thrombogenicity when compared with bare PDMS. Therefore, the results obtained in the current study suggest that the presence of the BS slightly suppresses the clotting process.

BSs have been reported to possess antiadhesive properties and are well known for their avoidance of microbial adhesion. Some studies have reported that hydrophilic surfaces tend to reduce the protein adsorption, thus interfering with the microbial adhesion process. ${ }^{11,12}$ Therefore, because the BSs are amphiphilic compounds and based upon the results from contact angle measurements, it seems that the

TABLE III. Chemical Composition Data by XPS of the Bare PDMS (control) and Biosurfactant-Modified PDMS

\begin{tabular}{lccccc}
\hline Elements & PDMS & Modification 1 & Modification 2 & Modification 3 & Modification 4 \\
\hline C1s & 44.3 & 48.7 & 43.7 & 43.2 & 50.5 \\
N1s & - & 4.06 & 3.63 & 31.2 & 5.58 \\
O1s & 26.4 & 26.9 & 29.7 & 21.6 & 25.6 \\
Si2p & 29.3 & 19.7 & 21.6 & 0.26 & 18.1 \\
Cl2p & - & 0.61 & 1.41 & 0.25 \\
\hline
\end{tabular}

Biosurfactant-modified PDMS: Modification 1, PDMS plus biosurfactant from Streptococcus thermophilus A; Modification 2, PDMS plus biosurfactant from Streptococcus thermophilus B; Modification 3, PDMS plus biosurfactant from Lactococcus lactis; Modification 4, PDMS plus biosurfactant from Lactobacillus paracasei. 


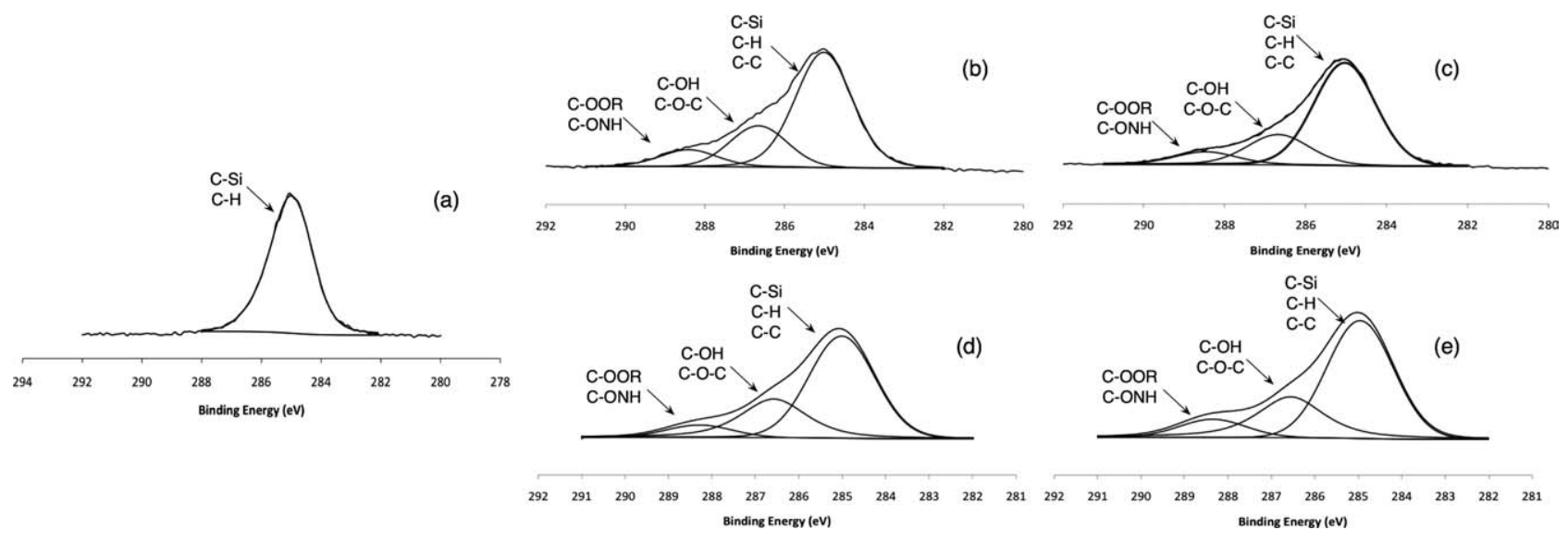

FIGURE 3. XPS spectra of C1s for (a) bare PDMS, PDMS modified with biosurfactants obtained from probiotic strains: (b) Streptococcus thermophilus A (modification 1), (c) Streptococcus thermophilus B (modification 2), (d) Lactococcus lactis (modification 3), and (e) Lactobacillus paracasei (modification 4).

BS molecules are oriented with their hydrophilic function toward the bulk. Hence, an antiadhesive effect of the modified PDMS surfaces could be expected. Indeed, an increase in the surface hydrophilicity was observed. Consequently, these modified surfaces would be expected to repel protein adsorption and avoid the thrombus formation. This is due to the fact that protein adsorption is the first step to initiating the coagulation cascade that ends with thrombus formation. $^{3}$ Nevertheless, it is important to notice that rough surfaces tend to increase the extent of protein adsorption and, as some roughness exists in the modified surfaces (Fig. 4), a dual effect might be expected. ${ }^{30}$
Furthermore, during the 40 min of contact, all the studied samples overcame the thrombogenicity on glass. This result leads to the conclusion that, although these modified PDMS surfaces perform better than the bare PDMS surface, they are not suitable for contact with blood, unless clotting is required. Results reported in others studies with a synthetic surfactant also indicated higher values of thrombosis. ${ }^{3}$

Hemolysis assay. The hemolysis results that were obtained for the bare and BS modified surfaces are shown in Figure 6.

Based upon the classification proposed by the ASTM F 756-00 standard and on the results from Figure 6, the bare
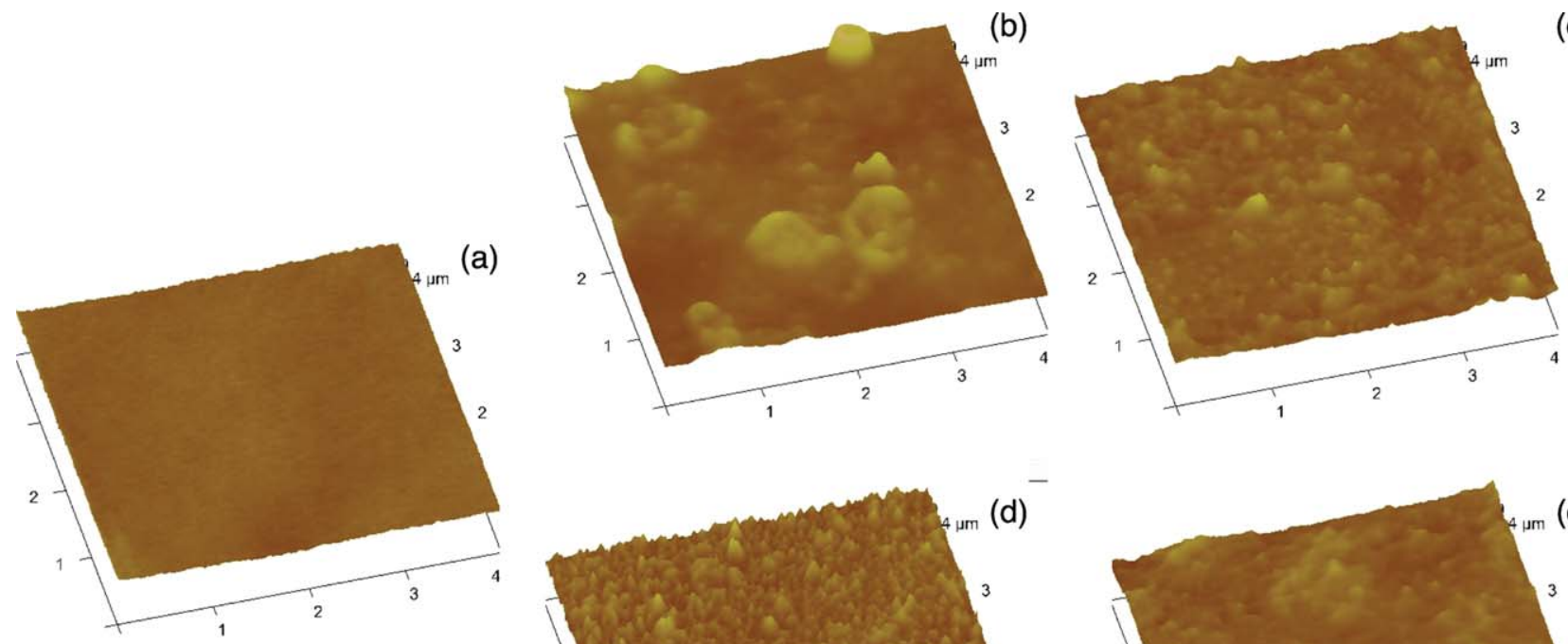

(c)
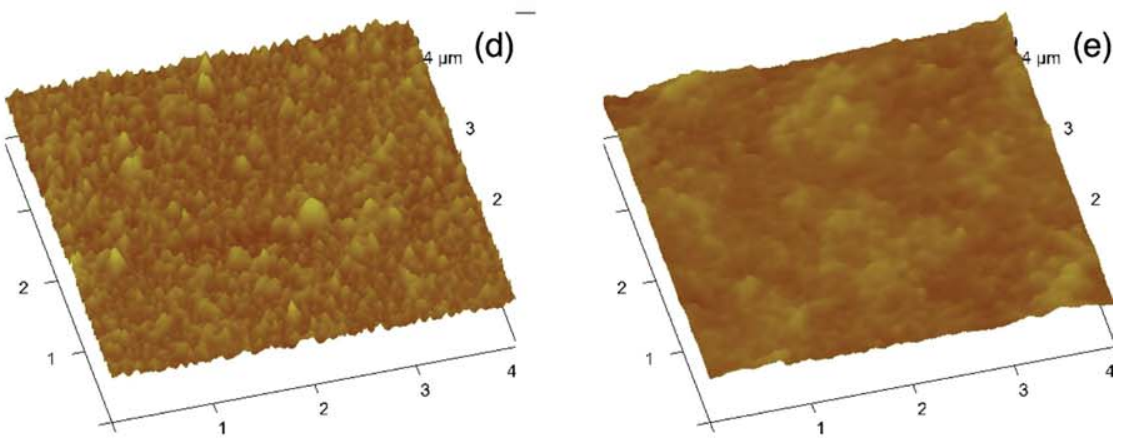

FIGURE 4. AFM micrographs of the (a) bare PDMS and PDMS modified with biosurfactants obtained from probiotic strains: (b) Streptococcus thermophilus A (modification 1), (c) Streptococcus thermophilus B (modification 2), (d) Lactococcus lactis (modification 3), and (e) Lactobacillus paracasei (modification 4). [Color figure can be viewed in the online issue, which is available at wileyonlinelibrary.com.] 


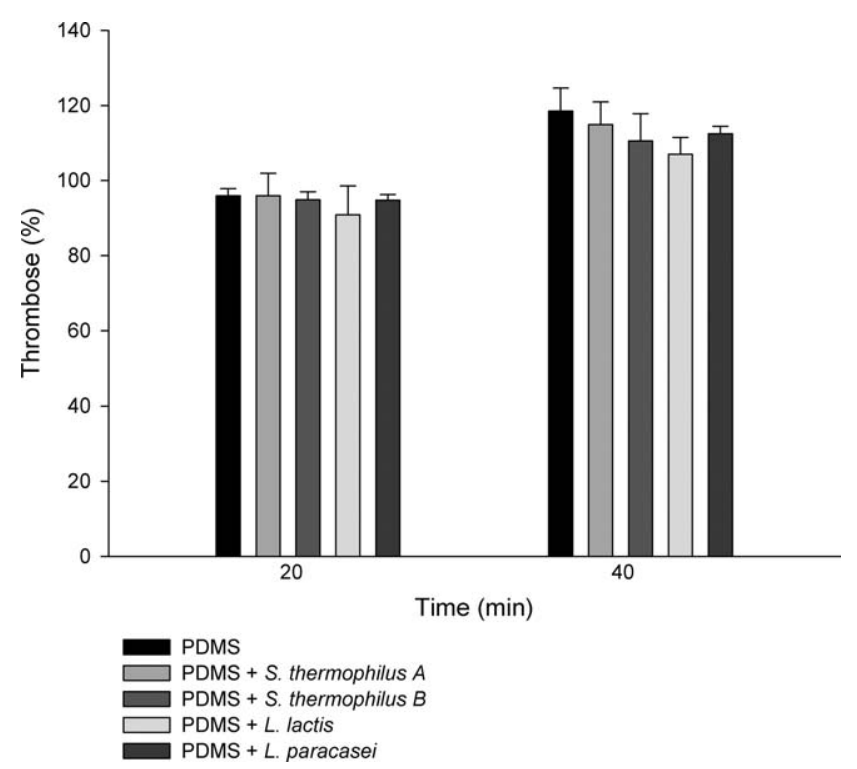

FIGURE 5. Thrombose values for bare PDMS and PDMS modified with biosurfactants from probiotic strains: Streptococcus thermophilus A (modification 1), Streptococcus thermophilus B (modification 2), Lactococcus lactis (modification 3), and Lactobacillus paracasei (modification 4), for 20 and 40 min of contact with blood. Results represent an average of three independent assays.

PDMS and PDMS modified with BSs gave only a very small hemolytic effect (hemolysis degree is less than $2 \%$ ). ${ }^{26}$ The modified PDMS surfaces gave lesser degrees of hemolysis comparing to the bare PDMS, due to the presence of BSs.

The red-cell membranes of erythrocytes, constituted by a phospholipid bilayer and small spots of proteins, are extremely fragile and deformable. When a membrane based on the cells is exposed to very hydrophobic surfaces, with a low surface energy, adhesion becomes favorable, and the

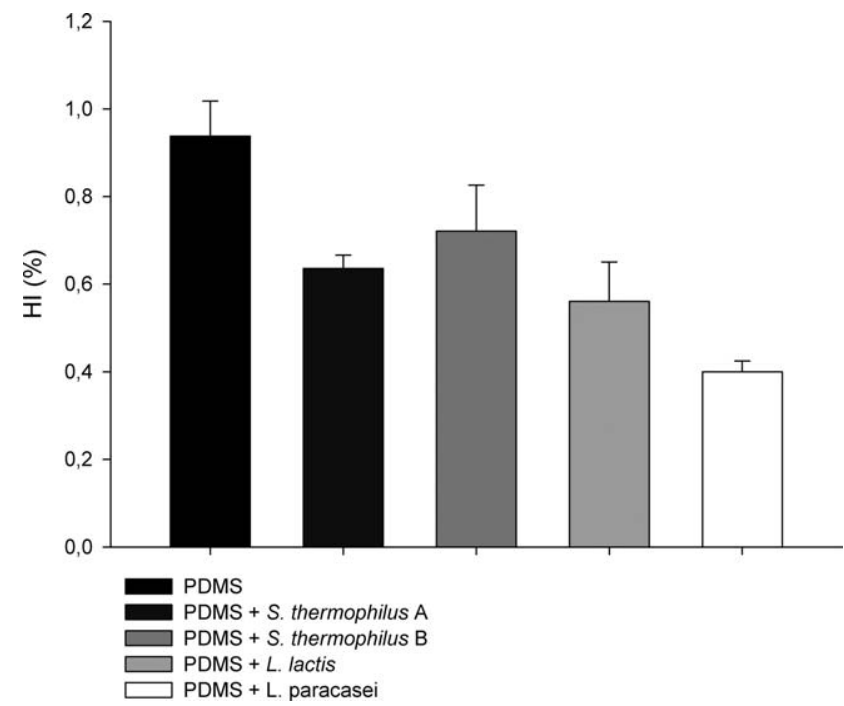

FIGURE 6. Hemolytic index for bare PDMS and PDMS modified with biosurfactants obtained from probiotic strains: $S$. thermophilus A (modification 1), Streptococcus thermophilus B (modification 2), L. lactis (modification 3), and L. paracasei (modification 4). Results represent an average of three independent assays. membrane becomes more fragile. Thus, the cell undergoes lysis, releasing hemoglobin. Hydrophilic surfaces have a weak interaction with red cells and, consequently, lysis is reduced. ${ }^{33}$ Therefore, the results obtained were what might have been expected, because the measured contact angles showed that the BS modified PDMS surfaces possessed a hydrophilic character. Furthermore, the hemolytic indexes, as determined in the current work, are in accord with the results of others reported studies, for example, with siloxane rubbers are nonhemolytic. ${ }^{34}$ Pinto and coworkers determined the hemolytic index for bare PDMS and for Pluronic ${ }^{\circledR}$ F-68 modified PDMS and obtained similar values for the haemolytic index of bare PDMS, but lower values than the ones that were obtained for BSs-modified PDMS. ${ }^{3}$ Accordingly, the modified PDMS surfaces were considered to be suitable for use in biomedical applications.

\section{Cytotoxycity assays}

In vitro cytotoxicity assays are limited in their scope due to their relative simplicity compared to the complex interactions occurring in vivo. Nevertheless, their ability to discriminate between biocompatible materials and nonbiocompatible materials can be useful in rejecting unsatisfactory materials, before in vivo implantation studies. ${ }^{35}$

Macrophages play a fundamental role in the control of the response of an organism to foreign substances/particles/devices. Macrophages are adherent cells in the immune system that respond to environmental aggressions. Because of these major features, they are widely used in viability and cytotoxicity studies to test new materials. ${ }^{27}$ However, knowledge concerning the influence of the chemical structure of the polymers on macrophage-implant recognition is scarce. ${ }^{36}$ Figure 7 presents the absorbance values (at $570 \mathrm{~nm}$ ) for the different BS modified PDMS, for 3 and

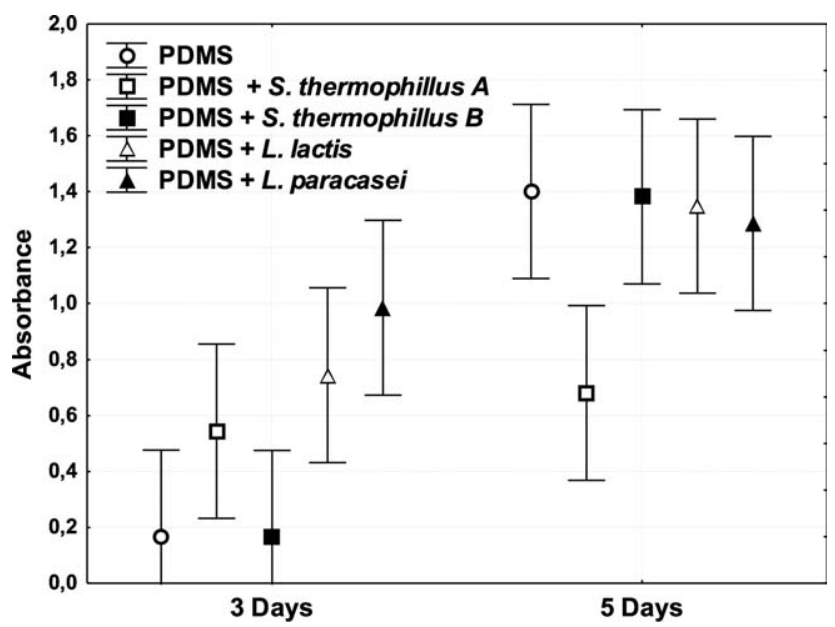

FIGURE 7. Cytotoxicity results for rats peritoneal macrophages along time ( 3 and 5 days) using MTT method, for bare and biosurfactant modified PDMS. Biosurfactants isolated from Streptococcus thermophilus A; Streptococcus thermophilus B; Lactococcus lactis; and Lactobacillus paracasei. Results correspond to an average of three independent assays. 
5 days of incubation. These absorbance values are representative of the number of viable cells, because the MTT assay enables the determination of metabolically active cells to be carried out meaningfully.

Significant differences were observed between all of the surfaces that were studied (bare PDMS and BS modified PDMS) after 3 days of incubation, $p<0.001$. Nevertheless, after 5 days of incubation, there are no significant differences, $p=0.122$. Furthermore, the coatings had a significant influence in the absorbance values of samples, from 3 to 5 days, $p<0.013$.

At an incubation time of 3 days, PDMS that was coated with the BS from $L$. paracasei presented the greatest absorbance value, suggesting that macrophages have a good interaction with this material. PDMS that was modified with BSs from L. lactis and from $S$. thermophilus A also showed good absorbance results, although slightly lower than those mentioned previously. After 5 days of incubation, the materials presented similar absorbance values, with the exception of $S$. thermophilus A. Bare PDMS and BS-modified PDMS (L. lactis, L. paracsei and S. thermophilus B) presented an increase of absorbance with time, which suggests a good and progressive adaptation of macrophages to the materials. Although a satisfactory performance of the macrophages was readily observed at the end of 3 days, for the PDMS that was modified with the BS from $S$. thermophilus A, there was no noteworthy increase in the cells activity afterward (at the end of 5 days).

According to the studies performed, the proposed structure of BSs that were obtained from L. lactis and L. paracasei is consistent with glycoproteins. ${ }^{14,15,17}$ This kind of structure has less $\mathrm{OH}$ groups than the glycolipid-like structure of the BSs obtained from $S$. thermophilus strains. ${ }^{16,31}$ The presence of $\mathrm{OH}$ groups, leading to the probable production of more oxygen-free radicals, is an important factor in the survival of the macrophages. Such radicals may induce polyunsaturated fatty acid peroxidation, leading to membrane damage. ${ }^{37}$

Nevertheless, cells incubated with bare PDMS or with PDMS that was coated with the $S$. thermophilus B BS gave a good recovery from 3 to 5 days, reaching identical values to the results from the L. lactis-coated PDMS and the L. paracasei-coated PDMS. Macrophages are very resistant cells. If they are in good condition, they can overcome from the suffered aggression. It has been suggested that glycolipids can interact with the migration inhibiting factor, enhancing their response after preincubation with this kind of molecule. ${ }^{38}$

Furthermore, macrophages incubated with PDMS that was coated with the $S$. thermophilus A BS gave a better result at first (3 days) but did not improve with time, contrarily to the behavior of $S$. thermophilus B. It has been shown, in vivo that, with some glycolipidic fractions, the inflammatory reaction has a peak at 6 days. ${ }^{39}$ The results may indicate that the two $S$. thermophilus strains correspond to different kinds of glycolipid. The B strain probably corresponds to a glycolipid with more $\mathrm{OH}$ groups (e.g., sphingoglycolipids) and/or different glucid residues, such as mannose and glucose residues. ${ }^{40}$
These results clearly show that both the incubation period and the type of BS, used to modify the PDMS surface, have an important and significant influence on the macrophages response. Furthermore, it is possible to conclude that all of the materials studied were noncytotoxic and suitable for use as biomaterials.

\section{CONCLUSIONS}

In this current work, it has been shown that the method used for PDMS surface modification was efficient with respect to improving some of its features, thus enabling its suitability for use in many biomedical applications. ATRFTIR and XPS analysis of the modified surfaces showed the formation of groups, species, and bonds that are not present in the bare PDMS. AFM characterization confirmed the change of the PDMS topography, suggesting the presence of a distinct layer at the surface corresponding to the BSs. The surface modifications conferred a hydrophilic character to the PDMS. Furthermore, the BS-modified surfaces were noncytotoxic and nonhemolytic. Therefore, the modified surfaces obtained in this study are potentially useful for several biomedical applications.

\section{REFERENCES}

1. Lee S, Vörös J. An aqueous-based surface modification of poly (dimethylsiloxane) with poly(ethylene glycol) to prevent biofouling. Langmuir 2005;21:11957-11962.

2. Zhang $X$, Lin G, Kumar SR, Mark JE. Hydrogels prepared from polysiloxane chains by end linking them with trifunctional silanes containing hydrophilic groups. Polymer 2009;50:5414-5421.

3. Pinto $S$, Alves $P$, Matos $C M$, Santos AC, Rodrigues LR, Teixeira JA, Gil MH. Poly(dimethyl siloxane) surface modification by low pressure plasma to improve its characteristics towards biomedical applications. Colloids Surf B 2010;81:20-26.

4. Chen H, Brook MA, Sheardown H. Silicon elastomers for reduced protein adsorption. Biomaterials 2004;25:2273-2282.

5. Zeraik $A E$, Nitschke $M$. Biosurfactants as agents to reduce adhesion of pathogenic bacteria to polystyrene surfaces: Effect of temperature and hydrophobicity. Curr Microbiol 2010;61:554-559.

6. Wu M. Simple poly(dimethylsiloxane) surface modification to control cell adhesion. Surf Interf Anal 2009;41:11-16.

7. Bodas D, Khan-Malek C. Formation of more stable hydrophilic surfaces of PDMS by plasma and chemical treatments. Microelectron Eng 2006;83:1227-1229.

8. Wang $\mathrm{P}$, Tan KL, Ho CC, Khew MC, Kang ET. Surface modification of natural rubber latex films by graft copolymerization. Eur Polym J 2000;36:1323-1331.

9. Makamba H, Kim JH, Lim K, Park N, Hahn JH. Surface modification of poly(dimethyl siloxane) microchannels. Electrophoresis 2003;24:3607-3619.

10. Bodas D, Malek CK. Hydrophilization and hydrophobic recovery of PDMS by oxygen plasma and chemical treatment-An SEM investigation. Sensor Actuat B: Chem 2007;123:368-373.

11. Meylheuc T, Methievier C, Renault M, Herry JM, Pradier CM, Fontaine NB. Adsorption on stainless steel surfaces of biosurfactants produced by gram-negative and gram-positive bacteria: Consequence on the bioadhesive behavior of Listeria monocytogenes. Colloids Surf B 2006;52:128-137.

12. Rodrigues L, Banat IM, Teixeira J, Oliveira R. Biosurfactants: Potencial applications in medicine. J Antimicrob Chemoth 2006; 57:609-618.

13. Singh $P$, Cameotra SS. Potential applications of microbial surfactants in biomedical sciences. Trends Biotechnol 2004;22:142-146.

14. Gudina E, Rocha V, Teixeira JA, Rodrigues LR. Antimicrobial and anti-adhesive properties of a biosurfactant isolated from Lactobacillus paracasei subsp. paracasei A20. Lett Appl Microbiol 2010; 50:419-424. 
15. Gudina E, Teixeira JA, Rodrigues LR. Isolation and functional characterization of a biosurfactant produced by Lactobacillus par acasei. Colloids Surf B 2010;76:298-304.

16. Rodrigues LR, Teixeira JA, Van de Mei HC Oliveira R. Isolation and partial characterization of a biosurfactant produced by Streptococcus thermophilus A. Colloids Surf B 2006;53:105-112.

17. Rodrigues LR, Teixeira JA, Van de Mei HC, Oliveira R. Physicochemical and functional characterization of a biosurfactant produced by Lactococcus lactis 53. Colloids Surf B 2006;49:78-85.

18. Chibowski E. Surface free energy of a solid from contact angle hysteresis. Adv Colloid Interf 2003;103:149-172.

19. DataPhysics Interfacial Chemistry, Operating manual DataPhysics OCA. Data Physiscs Instruments GmdH. Germany; 2002.

20. Shen W, Li Z, Liu Y. Surface chemical functional groups modification of porous carbon. Recent Patents Chem Eng 2008;1:27-40.

21. Tan HML, Fukuda H, Akagi T, Ichiki T. Surface modification of pol$y$ (dimethylsiloxane) for controlling biological cells' adhesion using a scanning radical microjet. Thin Solid Films 2007;515: 5172-5178.

22. Oehr C. Plasma surface modification of polymers for biomedical use. Nucl Instrum Meth B 2003;208:40-47.

23. Ferreira $P$, Pereira R, Coelho JFJ, Silva AFM, Gil MH. Modification of the biopolymer castor oil with free isocyanate groups to be applied as bioadhesive. Int J Biol Macromol 2007;40:144-152.

24. Besteiro MC, Guiomar AJ, Gonçalves CA, Bairos VA, Pinho MN Gil $\mathrm{MH}$. Characterization and in vitro hemocompatibility of bi-soft segment, polycaprolactone-based poly(ester urethane urea) membranes. J Biomed Mater Res A 2010;93:954-964.

25. ISO 10993-4: Biological Evaluation of Medical Devices, Part 4 Selection of Tests for Interaction with Blood. Geneva, Switzerland: International Organization for Standardization; 2002.

26. ASTM F 756-00-Standard Practice for Assessment of Hemolytic Properties of Materials. Philadelphia: American Society for Testing and Materials; 2000.

27. Santos AC, Matos CM, Cordeiro R, Brás AR, Oliveiros B, Campos $\mathrm{E}$, Alves $\mathrm{P}$, Rasteiro MG, Gil MH. Assessment of the cytotoxicity of microparticles for controlled drug release in oftalmology. Exp Pathol Health Sci 2008;2:33-37.
28. Weir DM. Handbook of Experimental Immunology, Vol. 2: Cellular Immunology. Oxford, UK: Blackwell Scientific; 1973. 2nd ed.

29. Bland M. An Introduction to Medical Statistics, 3rd ed. Oxford: Oxford University Press; 2000.

30. Strobel M, Lyons CS, Mittal, KL. Plasma Modification of Polymers: Relevance to Adhesion, Vol. 39. Netherlands: Polymer International; 1996.

31. Rodrigues LR, Van de Mei HC, Teixeira JA, Oliveira R. Biosurfactant from Lactococcus lactis 53 inhibits microbial adhesion on silicon rubber. Appl Genet Mol Biotechnol 2004;66:306-311.

32. Busscher HJ, Neu TR, Mei HC. Biosurfactant production by thermophilic dairy streptococci. Appl Microbiol Biotechnol 1984;41:4-7.

33. Masters KS. The haemocompatibility of polyurethane-hyaluronic acid copolymers. Biomaterials 2007;29:150-160.

34. Vijayanand K, Pattanayak DK, Mohan TRR, Banerjee R. Interpreting blood-biomaterial interactions from surface free energy and work of adhesion. Trends Biomater Artif Organs 2005;18:73-83.

35. Bélanger MC, Marois $\mathrm{Y}$. Hemocompatibility, biocompatibility, inflammatory and in vivo studies of primary reference materials low-density polyethylene and polydimethylsiloxane: A review. J Biomed Mater Res A 2001;58:467-477.

36. Smetana KJ, Vacík J, Houska M, Soucková D, Lucás J. Macrophage recognition of polymers: Effect of carboxylate groups. J Mater Sci: Mater Med 1993;4:526-539.

37. Abok K, Rundquist I, Forsberg B, Brunk U. Dimethylsulfoxide increases the survival and lysosomal stability of mouse peritoneal macrophages exposed to low-LET ionizing radiation and/or ionic iron in culture. Virchows Arch B Cell Pathol Incl Mol Pathol 1984; 46:307-320.

38. Liu DY, Yu SF, Miller A, Remold HG, David JR. Glycolipid-dependant interaction between human migration-inhibitory factor and mononuclear phagocytes. Cell Immunol 1984:88:350-360.

39. Silva CL, Foss NT. Inflammation induced by a glycolipid fraction from Mycobacterium leprae. Braz J Med Biol Res 1989;22:327-339.

40. StahI PD, Rodman JS, Miller MJ, Schlesinger PH. Evidence for receptor-mediated binding of glycoproteins, glycoconjugates, and lysossomal glycosidases by alveolar macrophages. Proc Natl Acad Sci USA 1978;75:1399-1403. 